\title{
6 Zusammenfassung und Ausblick
}

Die vorliegende Arbeit hat das Ziel verfolgt, sprachwissenschaftliche und kulturwissenschaftliche Auffassungen von Performativität mit einer soziologischen Theorie des Doing und Undoing zusammenzubringen, um das Phänomen des Namenwechsels transgeschlechtlicher Personen und den Zusammenhang von Vornamen und Geschlecht zu analysieren. Es ist deutlich geworden, dass Vornamen eine elementare Rolle in der Markierung von Geschlecht spielen, die von transgeschlechtlichen Personen genutzt werden, um ein Undoing des fremdzugewiesenen Geschlechts und ein Doing des tatsächlichen Geschlechts zu betreiben. Dabei steht weniger das Un/doing der Differenz Geschlecht im Vordergrund als das Un/Doing der jeweiligen Geschlechtskategorie.

Es wurde aufgezeigt, in welch vielfältigem Bezug Namen und Namenwechsel zu Geschlecht stehen und in welchem Umfang die transitionsbedingte Namenänderung zur Markierung weiterer sozialer Zugehörigkeiten wie Ethnizität und Religion genutzt wird. Darüber hinaus wurde deutlich, dass sowohl die Her- und Darstellung von Geschlecht am Namen als auch die Berücksichtigung anderer Differenzmarker dem primären Ziel dient, den eigenen Namen und damit die eigene Geschlechtlichkeit zu naturalisieren, um auf diese Weise eine möglichst unauffällige Geschlechtsnormalität zu leben und die Sichtbarkeit der eigenen Transgeschichte zu minimieren.

Durch die dem deutschen Namensystem inhärente Geschlechtsdefinitheit von Vornamen ist der Namenwechsel einer der salientesten Marker, um die geschlechtliche Transition zu kommunizieren und sichtbar zu machen. Abgesehen von der generellen Nutzung der kulturellen Geschlechtsklassifizierung von Namen wird Geschlecht vielfältig markiert, u. a. durch die Nachbenennung nach bestimmten Personen, die ein Geschlechtsideal verkörpern, oder durch die Wahl von Namen, mit denen bestimmte Geschlechtsattribute assoziiert werden. Äußerst wichtig bei der Entscheidung für einen neuen Namen ist dessen Geschlechtsirreversibilität, d. h. es werden Namen gewählt, die keine gegengeschlechtlichen Korrelate haben, um so die eigene geschlechtliche Positionierung gegen das fremdzugewiesene Geschlecht zu immunisieren.

Ferner ist auch die phonologische Geschlechtskodierung wichtig für die Wahrnehmung von und Entscheidung für Vornamen. Anhand des Gender-Indexes für Vornamen konnte gezeigt werden, dass Transfrauen und -männer unterschiedliche Strategien des phonologischen Doing Gender wählen: die Transfrauen, die an der Onlineumfrage teilgenommen haben, wählten überdurchschnittlich häufig Namen, die in späteren Generationen als der eigenen 
beliebt wurden und tendenziell femininere Lautstrukturen aufweisen. Da moderne Namen in der Wahrnehmung positiv mit Attraktivität korrelieren, ist dies als Doing Gender-Strategie zu interpretieren, die onymisch zur Stabilität des Geschlechts beitragen soll. Die stärkere Tendenz zu altersgerechten und der Gesamtbevölkerung entsprechenden Namen der Transmänner kann ebenso als eine Form von Doing Gender interpretiert werden, da solche Strategien der Namenwahl direkt an die alltagsweltlich als stärker traditionell verstandene Männerrolle anschließen, die sich in der Namengebung an Jungen widerspiegelt.

So sehr der enge Zusammenhang von Vornamen und Geschlecht von binärgeschlechtlich positionierten Transpersonen nutzbar gemacht wird, um sich vom fremdzugewiesenen Geschlecht zu distanzieren und die tatsächliche Geschlechtsmitgliedschaft zu markieren, so sehr bedeutet er für nichtbinäre Personen das Problem, dass sie in dem dichotomen Namen- wie Geschlechtssystem schlicht nicht vorgesehen sind. Daher stehen sie vor der Entscheidung, entweder einen stark markierten - da nicht im geläufigen Namenschatz vorhandenen - Namen zu wählen, oder aber sich für einen dem binären System entstammenden Namen zu entscheiden und ihr Geschlecht somit unsichtbar zu machen. Die Mehrheit der Teilnehmer_innen der Onlineumfrage und der Interviews wählte die letztgenannte Alternative, da ein Name, der nicht dem gewohnten Vornamenbestand entstammt, sowohl die transdifferente Positionierung unterstreicht und diese somit stets zum Thema macht, als auch einen offiziellen Namenwechsel nach TSG massiv erschwert und so in den meisten Fällen zu kontextsensitiver Mehrnamigkeit zwingt.

Neben der Namenwahl spielt der Zusammenhang von Vorname und Geschlecht auch in der sozialen Interaktion eine große Rolle, da die Verwendung des neuen Namens als Indikator für die Akzeptanz der Transgeschlechtlichkeit und geschlechtlichen Neupositionierung angesehen wird; wird der Name verweigert, führt dies häufig zum Kontaktabbruch mit Freund_innen und auch innerhalb der Familie. Nichtbinäre Personen müssen sich darüber hinaus damit auseinandersetzen, dass die „Echtheit“ ihrer Namen angezweifelt wird, wenn diese nicht dem typischen Personennamenbestand entsprechen. Sie müssen also neben ihrem Geschlecht auch den Namen selbst gegen den „Glauben“ anderer Personen behaupten, weshalb häufig auf die Durchsetzung des Namens in bestimmten, z. B. familiären, Kontexten verzichtet wird.

Die Studie hat weiterhin gezeigt, dass nicht nur die Geschlechterdifferenz bei der Wahl eines neuen Namens wichtig ist, sondern der Namenwechsel darüber hinaus genutzt wird, um weitere soziale Zugehörigkeiten auszudrücken. Dies dient häufig dem Zweck, insbesondere über die Markierung „vererbter“ Mitgliedschaften wie Ethnizität oder Religion familiale Zugehörigkeiten auszudrücken; 
auf diese Weise wird ein Doing Ethnicity/Religion while Doing Kinship betrieben. Innerfamiliäre Nachbenennung erfolgt häufig nach Vorfahr_innen länger zurückliegender Generationen, da die Namen entfernter Vorfahr_innen eine unbesetzte Projektionsfläche bieten, die Familialität ermöglicht, ohne die eigene Individualität einzuschränken. Gleichermaßen kann die Wahl eines neuen Namens auch als explizite Zurückweisung von im Geburtsnamen markierten Differenzen wirken. Auf diese Art kann nicht nur die entsprechende ethnische/religiöse/regionale Zugehörigkeit, sondern auch die damit einhergehende familiale Anbindung negiert werden.

Sowohl die Markierung familialer als auch kategorialer Zugehörigkeiten hat eine stark naturalisierende und normalisierende Wirkung; gerade die onymische Markierung von Differenzen, die auch durch andere Zeichen indiziert werden (Hautfarbe, Kopftuch, Dialekt/Akzent etc.), trägt dazu bei, den Namen glaubhaft als von den Eltern zum Zeitpunkt der Geburt vergeben erscheinen zu lassen, was gleichzeitig zur Glaubhaftigkeit des Geschlechts beiträgt. Hier werden also andere soziale Zugehörigkeiten in den Dienst der Geschlechtsmarkierung gestellt, die weiter profiliert wird. Auch die Einbeziehung der Eltern in die Wahl des Namens, die bis zur Rückgabe des Benennungsrechts an sie reicht, naturalisiert den „Sonderfall Neubenennung“ und stellt diesen so auf eine vergleichbare Ebene mit der bei der Geburt erfolgten Namenvergabe. Die onymische Berücksichtigung von Eltern und familialer Zugehörigkeit ist außerdem in vielen Fällen eine bewusste Strategie, soziale Beziehungen wie das Eltern-KindVerhältnis zu verhandeln und innerhalb dieser Beziehungen die Akzeptanz des Namenwechsels und der Transgeschlechtlichkeit zu erhöhen.

Neben dem Vornamen sind die Personalpronomen das wichtigste Zeichen sprachlicher Geschlechtsmarkierung. Die Studie zeigt deutlich, dass die Verwendung des neuen Pronomens deutlich mehr Zeit in Anspruch nimmt und sehr viel fehleranfälliger ist als die des Namens. Diese größere Schwierigkeit der neuen Pronominalisierung lässt sich auf die Monofunktionalität des Pronomens - im Gegensatz zur Bifunktionalität des Namens - zurückführen: während der Name stets - und manchmal ausschließlich - individualisiert und dabei von Geschlecht absehen kann, ist das Pronomen pure geschlechtliche Referenz, d. h. es ist semantisch völlig leer. Die individualisierende, nicht geschlechtszuweisende Referenzfunktion des Namens fällt vielfach leichter als die des Pronomens, das exklusiv vergeschlechtlicht, ohne den Ausweg in die individuierende Verwendung zu bieten. Gerade im Kontext der falschgeschlechtlichen Pronominalisierung wurde deutlich, wie wenig eine Person selbst über ihr eigenes sprachliches Geschlecht bestimmen kann und wie stark die eigene geschlechtliche Positionierung davon abhängig ist, interaktional in diesem Geschlecht platziert zu werden. Zwar kann man die Adressierung in einem Geschlecht einfordern, ob dem jedoch 
von anderen Folge geleistet wird, muss deren Bereitschaft, dieses Geschlecht zu „glauben“ bzw. die geschlechtliche Selbstpositionierung anderer zu respektieren, überlassen bleiben.

Auch hinsichtlich der Pronominalisierung befinden sich nichtbinäre Personen in einer besonders komplexen Situation: Aufgrund der Inexistenz einer verbreiteten Form für die geschlechtsneutrale Pronominalisierung in der 3. Person Singular - vergleichbar zu schwed. hen oder engl. they - müssen sie sich entscheiden, ihre geschlechtliche Positionierung sprachlich unsichtbar $\mathrm{zu}$ machen und die Referenz mit er oder sie zu akzeptieren, oder durch Verwendung von und Bitte um neutrale Pronomenformen wie sier, xier oder $a$ ihr Geschlecht kontinuierlich $\mathrm{zu}$ thematisieren und sich dadurch beständig in eine Position exponierter Sichtbarkeit zu begeben. Nichtbinäre Personen, die ihr Geschlecht sprachlich markieren, befinden sich so in einer Position der Transdifferenz, die sie nicht als „Transitraum“ begreifen, sondern als dauerhaften Ort geschlechtlicher Selbstverortung, die sich nicht in ein binäres System einhegen lässt. Die Schwierigkeiten nichtbinärer Personen mit Vornamen und Pronomen zeigen deutlich, dass die deutsche Gegenwartsprache (noch) keinen linguistischen Raum für nichtbinäre Positionierungen vorsieht.

Die Markierung von Geschlecht ist nicht Namen und Pronomen allein vorbehalten, sondern wird ebenso durch eine Vielzahl anderer Geschlechtsmarker geleistet. Die Interaktion dieser Marker wird, wie in den Interviews deutlich wurde, detailliert reflektiert und bei der Transition bzw. der Publikation des neuen Namens sorgfältig berücksichtigt. Dies gilt insbesondere für optische Marker. Es wird weitgehend versucht, Widersprüchlichkeit zwischen den verschiedenen Markern $\mathrm{zu}$ vermeiden, indem neue Namen erst dann mitgeteilt werden, wenn das Aussehen als ausreichend dem tatsächlichen Geschlecht entsprechend eingeschätzt wird. Gleichzeitig wird der Name auch genutzt, um ein potenziell ambiges Äußeres zu vereindeutigen; die Erwähnung des eigenen Namens trägt dazu bei, andere Marker für das Umfeld verstehbar zu machen. Die Transition kann so als ein semiotischer Kongruierungsprozess beschrieben werden, bei dem mehrere Marker gleichzeitig manipuliert werden, um als das tatsächliche Geschlecht für andere wahrnehmbar zu werden.

Es wurde deutlich, dass verschiedene Marker eine unterschiedliche Bedeutung für Transmänner und Transfrauen haben, insbesondere in Hinblick auf die Stimme: Für viele Transmänner, die hormonell transitionieren, wird die Stimme zu einem wichtigen Geschlechtsmarker, da sie ebenso wie Cismänner in der Pubertät einen Stimmbruch durchlaufen, und eine tiefe Stimme einen wichtigen Ausgleich zu weniger direkt indizierenden Geschlechtsmerkmalen bilden kann. Dagegen ändert sich die Stimme von Transfrauen, die in ihrer Vergangenheit einen Stimmbruch durchlaufen haben, trotz hormoneller 
Transition nur geringfügig; sie bleiben daher häufig durch ihre Stimme im fremdzugewiesenen Geschlecht verhaftet, sodass die Bedeutung optischer Geschlechtsmarker zunimmt, die den potenziell gegengeschlechtlichen Effekt der Stimme nivellieren bzw. ihre geschlechtliche Markerfunktion herabstufen können. Gleichzeitig wurde deutlich, dass für Transfrauen sowohl in Hinsicht auf onymische als auch optische Marker die Angst vor einem Overdoing Gender besteht, das ihre geschlechtliche Positionierung stärker bedrohen kann als dies für Transmänner der Fall ist.

Dass die Stabilität der geschlechtlichen Positionierung nicht nur von der Interaktion sprachlicher, stimmlicher und visueller Marker abhängig ist, hat sich darin gezeigt, wie wichtig die Änderung des Namens auf offiziellen Ausweisdokumenten ist. Der alte Name auf personalisierten Fahrkarten, EC-Karten u.ä. führt nicht nur potenziell zum Zwangsouting, sondern kann bei zu großer Diskrepanz Beschuldigungen kriminellen Handelns nach sich ziehen. Der derzeitige Mangel an offiziellen Richtlinien, wie in Universitäten, Banken, Krankenversicherungen etc. mit Namenänderungen, die (noch) nicht rechtlich anerkannt sind, umzugehen ist, führt zu uneinheitlicher Handhabung. Dies ist insbesondere dann problematisch, wenn die rechtliche Namenänderung nach TSG nicht möglich ist bzw. nicht angestrebt wird, weil das Verfahren zu kostspielig ist, die Begutachtungspflicht abgelehnt wird oder die dem Gesetz zugrundeliegende binäre Geschlechtslogik keinen Raum für die eigene geschlechtliche Positionierung bietet. Die derzeitige Fassung des TSG schafft so vielfältige Ausschlüsse, die bedeuten, dass einer Vielzahl an Personen eine offizielle Änderung ihres Namens verunmöglicht wird und somit eine Kongruenz zwischen Ausweispapieren, Optik und inoffiziell geführten Namen verwehrt bleibt. Es bleibt abzuwarten, ob und in welchem Umfang die Änderung des Personenstandsgesetzes im Zuge der Einführung der dritten Geschlechtsoption und die Bemühungen um eine Reform des TSG zu einer nachhaltigen Veränderung der derzeitigen unzureichenden rechtlichen Berücksichtigung transgeschlechtlicher Personen beitragen werden.

Insgesamt hat die Studie verdeutlicht, dass die Wahl des neuen Namens ebenso wie die Kommunikation des Namens und Pronomens allem voran von dem Wunsch nach Unauffälligkeit und Geschlechtsnormalität geprägt ist, in deren Dienst die onymische Markierung von Geschlecht und anderen sozialen Differenzen gestellt wird. Das primäre Ziel im Umgang mit dem Namenwechsel ist ein Doing Normality und Doing Inconspicuousness; die Wahl des neuen Namens und die bewusste Entscheidung für die damit einhergehende Markierung spezifischer sozialer Differenzen kann so als Passingtechnik interpretiert werden, durch die die eigene Transitionsgeschichte $\mathrm{zu}$ Geschichte und das Präfix trans- vor dem eigenen Geschlecht gestrichen werden soll. Der neue Name wird, ebenso wie andere Marker wie Kleidung oder Gestik, zu einem Instrument 
der Geschlechtsnormalität, das ein möglichst unbehelligtes Leben im tatsächlichen Geschlecht ermöglichen soll.

Durch die kulturhistorische und politische Einschreibung der Geschlechtsdifferenz in das Vornamensystem ist ein Undoing Gender im Sinne einer Herabstufung der Relevanz dieser Differenz auf individueller Ebene kaum möglich; das Wissen um die inhärente Geschlechtlichkeit von Vornamen führt unvermeidlich zur geschlechtlichen Interpretation eines Namens. Das Un/Doing Masculinity/ Femininity, das binärgeschlechtliche Transpersonen am Namen betreiben, dient primär der Auflösung einer transdifferenten Positionierung, die durch die Inkongruenz von Geschlechtsmarkern entsteht, und verfolgt das Ziel, ein Undoing Transness zu bewirken. Ebenso führt das von vielen nichtbinären Personen angestrebte Undoing Gender am Vornamen nicht zu einem Undoing im Hirschauer'schen (2016) Sinn des Nicht-Stattfindens dieser Differenz, sondern zu einem Doing Gender Differently, das eher Butlers (2004) Verständnis von Undoing Gender entspricht. Statt von Undoing Gender lässt sich hier im Anschluss an Breinig/Löschs $(2002,2010)$ Transdifferenz-Konzept von einem Undoing Binarity sprechen, das die Geschlechtsdichotomie herausfordert und versucht, Positionierungen außerhalb zu etablieren, die das Potenzial haben, auf längere Sicht zu einem Relevanzverlust der Geschlechtsmarkierung am Namen beizutragen.

Die in dieser Arbeit vorgestellte Studie ist die erste, die sich ausführlich mit der Namenwahl transgeschlechtlicher Personen auseinandergesetzt hat. Sie hat aufgezeigt, wie lohnenswert es ist, das selbstverständliche Übersehen der sozialen Dimension von Namen und Benennungspraktiken, das in der Wissenschaft vorherrscht, herauszufordern und sie zum Gegenstand akademischer Auseinandersetzung zu machen. Dadurch wird es möglich, die Funktion des Namens zur Markierung sozialer Positionierungen zu analysieren und die onymischen Praktiken des Doing Differences in den Blick zu nehmen. Darüber hinaus stellt die Studie den enormen Stellenwert des Namens und der in ihm markierten sozialen Informationen für die tagtägliche zwischenmenschliche Interaktion heraus und zeigt die ordnende Funktion des Vornamens für die Interpretation der vergeschlechtlichten sozialen Welt auf.

Selbstverständlich kann diese Arbeit nicht alle Fragestellungen befriedigend und umfänglich beantworten. So sind z. B. künftige Studien zum Doing Gender von Vornamen wünschenswert, die den phonologischen Gender-Index weiter ausbauen und die kulturelle Dimension der Geschlechtsinformation von Vornamen näher beleuchten. Ebenso wären Studien zum Namenwechsel nicht-transgeschlechtlicher Personen gewinnbringend, um herauszustellen, wie ähnlich oder verschieden Strategien des Doing Differences mittels Namen tatsächlich sind. Darüber hinaus dürften komparative Studien, die die Benennungspraktiken und den Umgang mit Namenänderungen transgeschlechtlicher 
Personen in Ländern mit liberalerer Namengesetzgebung zum Vergleich heranziehen, sehr fruchtbar sein. Weiterhin werden künftige Studien äußerst erhellend sein, die darstellen, wie die derzeit stattfindenden Änderungen im Personenstandsrecht und die angestrebte Reform des TSG die Situation transgeschlechtlicher Personen generell sowie spezifisch bezüglich des Umgangs mit Namenwahl und -verwendung verändern werden. 
